\title{
Drug screening and identification of key candidate genes and pathways of rheumatoid arthritis
}

\author{
YU-QUAN SHI, WU-FANG QI and CHUN-YU KONG \\ Department of Rheumatology and Immunology, Tianjin First Central Hospital, Tianjin 300192, P.R. China
}

Received September 21, 2019; Accepted April 22, 2020

DOI: $10.3892 / \mathrm{mmr} .2020 .11168$

\begin{abstract}
Rheumatoid arthritis (RA), which normally manifests as a multi-joint inflammatory reaction, is a common immunological disease in clinical practice. However, the pathogenesis of RA has not yet been fully elucidated. Rituximab (RTX) is an effective drug in the treatment of RA, however its therapeutic efficacy and mechanism of action require further investigation. Thus, the present study aimed to screen the candidate key regulatory genes and explain the potential mechanisms of RA. Gene chips of RA and normal joint tissues were analyzed and, gene chips of RTX before and after treatment were investigated. In the present study, strong evidence supporting the pathogenesis of RA and mechanism of action of RTX were also revealed. Differentially expressed genes (DEGs) were analyzed using the limma package of RStudio software. A total of 1,150 DEGs were detected in RA compared with normal joint tissues. The upregulated genes were enriched in 'interleukin-12 production', 'I- $\kappa \mathrm{B}$ kinase/NF- $\mathrm{B}$ signaling', 'regulation of cytokine production involved in immune response' and 'cytokine metabolic process'. Functional enrichment analysis showed that RTX was primarily involved in the inhibition of 'adaptive immune response', 'B cell activation involved in immune response' and 'immune effector process'. Subsequently, leukocyte immunoglobulin-like receptor subfamily B member 1 (LILRB1), a hub gene with high connectivity degree, was selected, and traditional Chinese medicine libraries were molecularly screened according to the structure of the LILRB1 protein. The results indicated that kaempferol 3-O- $\beta$-D-glucosyl- $(1 \rightarrow 2)-\beta$-D-glucoside
\end{abstract}

Correspondence to: Professor Chun-Yu Kong, Department of Rheumatology and Immunology, Tianjin First Central Hospital, 24 Fu-Kang Road, Nankai, Tianjin 300192, P.R. China

E-mail: kongchunyu@aliyun.com

Abbreviations: RA, rheumatoid arthritis; LILRB1, leukocyte immunoglobulin-like receptor subfamily B member 1; DEGs, differentially expressed genes; PPI, protein-protein interaction; GO, Gene Ontology; KEGG, Kyoto Encyclopedia of Genes and Genomes; TCM, Traditional Chinese Medicine

Key words: rheumatoid arthritis, rituximab, LILRB1, kaempferol, bioinformatic analysis exhibited the highest docking score. In the present study, the DEGs and their biological functions in RA and the pharmacological mechanism of RTX action were determined. Taken together, the results suggested that LILRB1 may be used as a molecular target for RA treatment, and kaempferol 3-O- $\beta-\mathrm{D}$ -glucosyl-( $1 \rightarrow 2)-\beta-D$-glucoside may inhibit the pathological process of RA.

\section{Introduction}

Rheumatoid arthritis (RA) is a chronic systemic disease accompanied by inflammatory synovitis that is mainly characterized by symmetrical distribution of invasive joint inflammation of the hand and foot $(1,2)$. In addition, RA exhibits increased interstitial inflammatory cell infiltration and bone tissue destruction, resulting in joint deformity and loss of function (3). Immune function is considered to be the main aspect associated with RA; RA is characterized by the induction of innate immune disorders, including immune complex-mediated complement activation, osteoclast and chondrocyte activation and cytokine network dysregulation, which develop semi-autonomous features that contribute to disease progression $(4,5)$. However, the exact mechanism of RA development remains elusive and further investigation is required.

General, surgical and pharmaceutical therapies are widely applied in RA treatment (6). The most commonly used pharmacological RA drugs include the administration of non-steroidal anti-inflammatory drugs, immunosuppressants, botanicals and biological agents (7). Rituximab (RTX), a chimeric monoclonal antibody against the CD20 ligand of B lymphocytes, has been reported to exhibit therapeutic activity in the clinical treatment of RA (8); however, its therapeutic mechanism needs to be further investigated. Although several drugs alleviate pain in patients with RA, their efficacy is limited (9), therefore the development of novel and effective drugs for RA is required.

The present study aimed to further elucidate the pathogenesis of RA and identify potential drugs for RA treatment. The expression profiles of normal, RA control and RTX-treated tissues were analyzed. A series of immune-related genes, including leukocyte immunoglobulin-like receptor subfamily B member 1 (LILRB1), were detected by screening the differentially expressed genes (DEGs). The results revealed that LILRB1 was associated with RA pathogenesis. LILRB1, 
an inhibitory receptor broadly expressed in leukocytes, has been demonstrated to regulate immune responses by binding to MHC class I molecules on antigen-presenting cells (10). Finally, Traditional Chinese Medicine (TCM) libraries were molecularly screened for this key functional gene in order to identify potential therapeutic drugs.

\section{Materials and methods}

Download of expression profile chip data and DEGs analysis. The screening of DEGs $(11,12)$ in the synovial tissues of normal patients without RA and patients with RA (GSE55235) (13) was performed using the Gene Expression Omnibus (GEO) database (14) and differential gene analysis. In addition, DEG screening in RA and RTX-treated patients (GSE24742) (15) was assessed using the GEO database and $\mathrm{R}$, version 3.6.2. Data quality was determined by calculating residual sign, residuals, weight, relative log expression, normalized unscaled standard errors and RNA degradation. Finally, the differences in RNA expression profiles between groups were analyzed using the pheatmap and limma $\mathrm{R}$ packages $(16,17) . \mathrm{ILog}_{2}$ fold-change (FC) $\mid \geq 1$ and $\mathrm{P}<0.05$ were set as the cutoff criteria for DEGs.

Gene Ontology (GO) and Kyoto Encyclopedia of Genes and Genomes (KEGG) analyses $(18,19)$. The functions of DEGs were analyzed using the ClueGO plug-in application in Cytoscape 3.6.1 (https://cytoscape.org). In addition, KEGG pathway enrichment analysis (20) was carried out using ClueGO (21) and visualized using CluePedia (22). $\mathrm{P}<0.05$ was set as the cutoff value.

Gene set enrichment analysis (GSEA). GSEA analysis was performed using the GSEA software (23). In brief, the method consisted of the following steps. First, the gene data, including expression profiles and class distinctions, were listed. Given a defined set of genes, the goal of GSEA was to determine whether the members of the gene set were found at the top or bottom of the list. Subsequently, an enrichment score was calculated in order to identify the degree of the over-represented genes. Finally, a weighted enrichment statistics was carried out and the number of random permutations was set to 1,000 times.

Pathway analysis. DEGs were subjected to signal pathway enrichment analysis using the ConsensusPathDB database (http://cpdb.molgen.mpg.de) $(24,25)$. The files containing the measured genetic data were uploaded to the database and subsequently pathway enrichment analysis was carried out using the gene set analysis function.

Protein-protein interaction (PPI) analysis. The PPI network was analyzed using STRING software (https://string-db. org) (26). The organism selected was 'Homo sapiens'. The interacting protein complexes that functionally influence the physiological processes of a disease and the PPI enrichment analysis reflected the interaction between DEGs.

TCM database and molecular docking simulation of LILRB1. A total of 32,364 compounds were obtained from the TCM database (http://tcm.cmu.edu.tw) (27). The conformational energy of small molecules was minimized using the Maestro software (version 11.8, Schrödinger, LLC) by adding hydrogen atoms and removing counter ions and salts $(28,29)$. The crystal structure of LILRB1 was downloaded from Protein Data Bank (structure no. 1UGN; http://www.rcsb.org) (30) and the protein structure was refined by removing crystalline water and ions. In addition, energy minimization was performed on the LILRB1 protein structure. TCM docking and the selection of candidate compounds was performed using the virtual screening workflow model of Schrodinger and Glide XP (extra precision), respectively (31-33).

\section{Results}

Identification of DEGs in RA. The gene expression profiles of the synovial tissues of patients with RA from GSE55235 were obtained from the GEO database. The microarray data from GSE55235 included synovial tissues from 10 healthy and 10 RA joints. A total of 1,150 DEGs were extracted from the expression profile data set, including 508 downregulated and 642 upregulated genes. $\mid \log _{2} \mathrm{FCl} \geq 1$ and $\mathrm{P}<0.05$ were set as the cutoff criteria for DEGs. The distribution of DEGs is presented in a volcano plot (Fig. 1A). In addition, hierarchical cluster analysis was performed in order to obtain an overview of the expression profiles of normal and RA tissues (Fig. 1B). Finally, all heat maps demonstrated different adjustment directions and significant separation between normal and RA samples.

GO and KEGG analyses of DEGs in RA. GO and KEGG enrichment analyses of DEGs were performed using the ClueGO plug-in in Cytoscape software, and subsequently the upregulated and downregulated genes were analyzed. The enriched upregulated genes were mainly associated with 'interleukin-12 production', 'I- $\kappa \mathrm{B}$ kinase/NF- $\kappa \mathrm{B}$ signaling', 'regulation of cytokine production involved in immune response', 'positive regulation of B cell differentiation', 'cytokine metabolic process' and 'activation of immune response' (Fig. 2A). The results of the pathway analysis showed that RA mainly affected the 'Fc $\gamma$ R-mediated phagocytosis', 'natural killer cell-mediated cytotoxicity', 'B cell receptor signaling pathway', 'NF-кB signaling pathway' and 'leukocyte transendothelial migration' (Fig. 2B). By contrast, the downregulated genes were mainly involved in 'vasculogenesis', 'regulation of DNA binding transcription factor activity', 'cellular response to external stimulus', 'vascular process in circulatory system', 'blood circulation' and 'transcription factor binding' (Fig. 3A). Finally, the pathway enrichment analysis indicated that the downregulated DEGs were mainly enriched in 'tyrosine metabolism', 'FoxO signaling pathway', 'regulation of lipolysis in adipocytes' and 'colorectal cancer' (Fig. 3B).

Identification of DEGs based on RTX treatment data. A total of 54,675 genes were obtained from 12 control and 12 RTX-treated samples, and 941 DEGs (382 upregulated and 559 downregulated) were identified. The volcano plot of DEGs is presented in Fig. 4A. The red, green and black dots indicate the upregulated, downregulated and non-differentiated genes, respectively. Finally, the overview of the expression profiles 

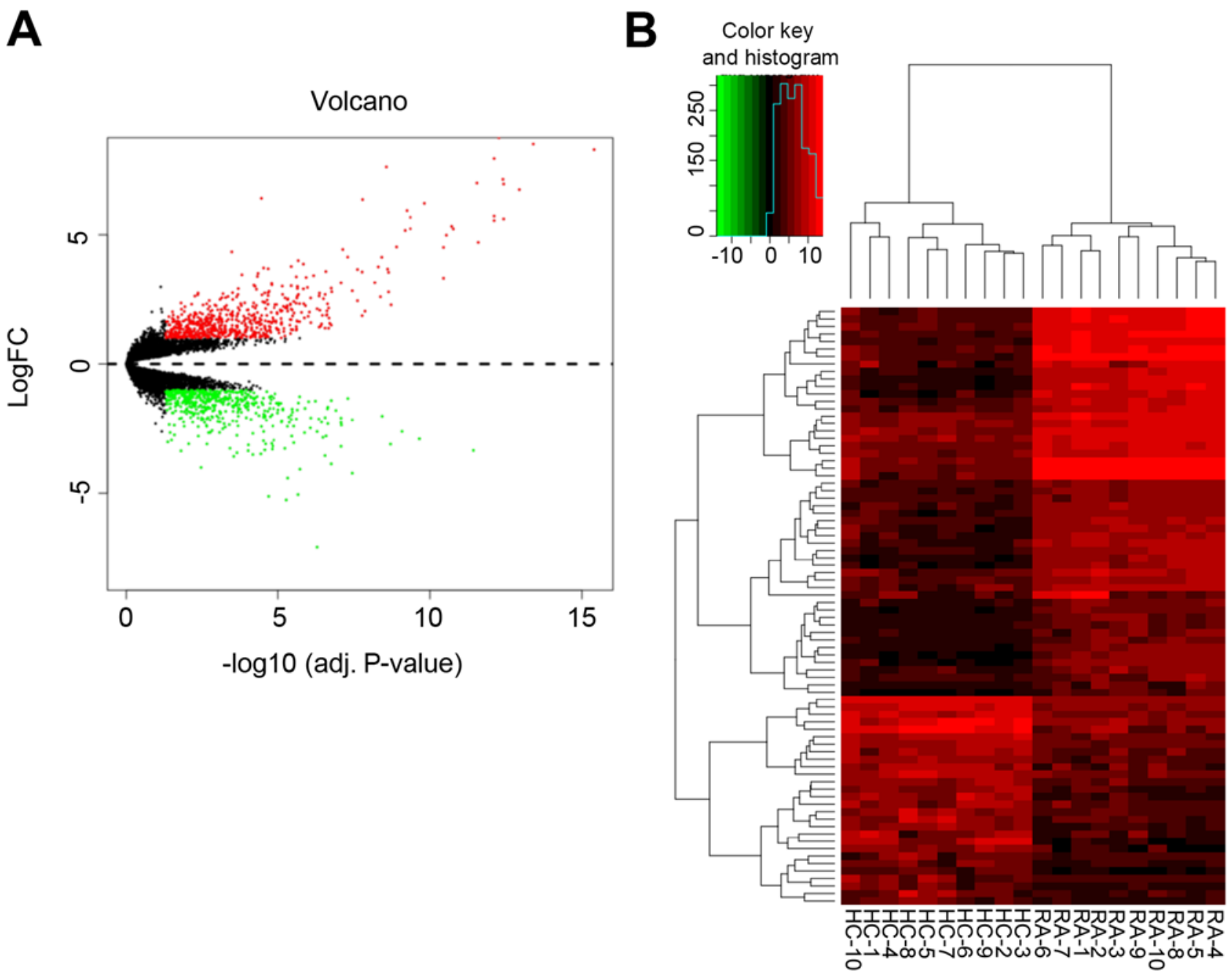

Figure 1. Analysis of differentially expressed genes between normal and rheumatoid arthritis joint synovial tissue samples. (A) Volcanic map of genes between normal and rheumatoid arthritis joint synovial tissue samples. Red and green dots indicate the upregulated and downregulated genes, respectively. The black dots correspond to gene expression with $\log _{2} \mathrm{FCl}<1$. The $\mathrm{x}$-axis represents false discovery rate and the y-axis denotes the value of $\log _{2} \mathrm{FC}$. (B) Cluster analysis results based on the expression profiles of differentially expressed genes. FC, fold-change.

of DEGs before and after RTX treatment (Fig. 4B), and the distribution of genes between groups were revealed using a hierarchical cluster analysis.

GSEA and pathway enrichment analyses of DEGs after $R T X$ treatment. GSEA was performed in order to further confirm the selected DEGs from the GO functional enrichment analysis (Fig. 5A). GSEA revealed that RTX upregulated the expression of genes associated with the 'positive regulation of dendritic development' and the 'regulation of neuron migration'. By contrast, the 'adaptive immune response', 'anaphase-promoting complex-dependent catabolic process', 'antigen processing and presentation', 'B cell activation involved in immune response' and 'immune effector process' functions were suppressed. Additionally, pathway analysis was performed, using the online ConsensusPathDB database, in order to analyze the functional and signaling pathway enrichment of the gene signatures (Fig. 5B).

The pathway enrichment analysis also revealed that the downregulated DEGs were enriched in the 'PI3K-Akt signaling pathway', 'type II interferon signaling', 'Janus kinase/STAT signaling pathway', 'interleukin-6 signaling pathway', 'T helper 17 cell differentiation', and 'interleukin-4 and interleukin-13 signaling'. The aforementioned results supported the conclusion that RTX may treat RA via regulating body immunity.

Identification of key candidate genes using STRING protein interaction network. A Venn diagram analysis of the upregulated and downregulated genes in RA and after RTX treatment groups, respectively, was performed (34). The analysis identified 13 key genes that were subsequently analyzed using the STRING database (http://string-db.org) for PPI network analysis (Fig. 6A). The results indicated that LILRB1 exhibited the highest interactivity confidence (Fig. 6B).

Screening of candidate compounds for RA treatment. The PPI network analysis indicated that LILRB1 was a key node gene associated with the mechanisms of RA pathogenesis. Therefore, the LILRB1 gene was selected for virtual drug screening. A set of molecular recognition strategies for TCM compounds identification was designed via structure-based high-throughput virtual screening. The filtering process is 
A

Gene Oncology enrichment analysis of upregulated gene

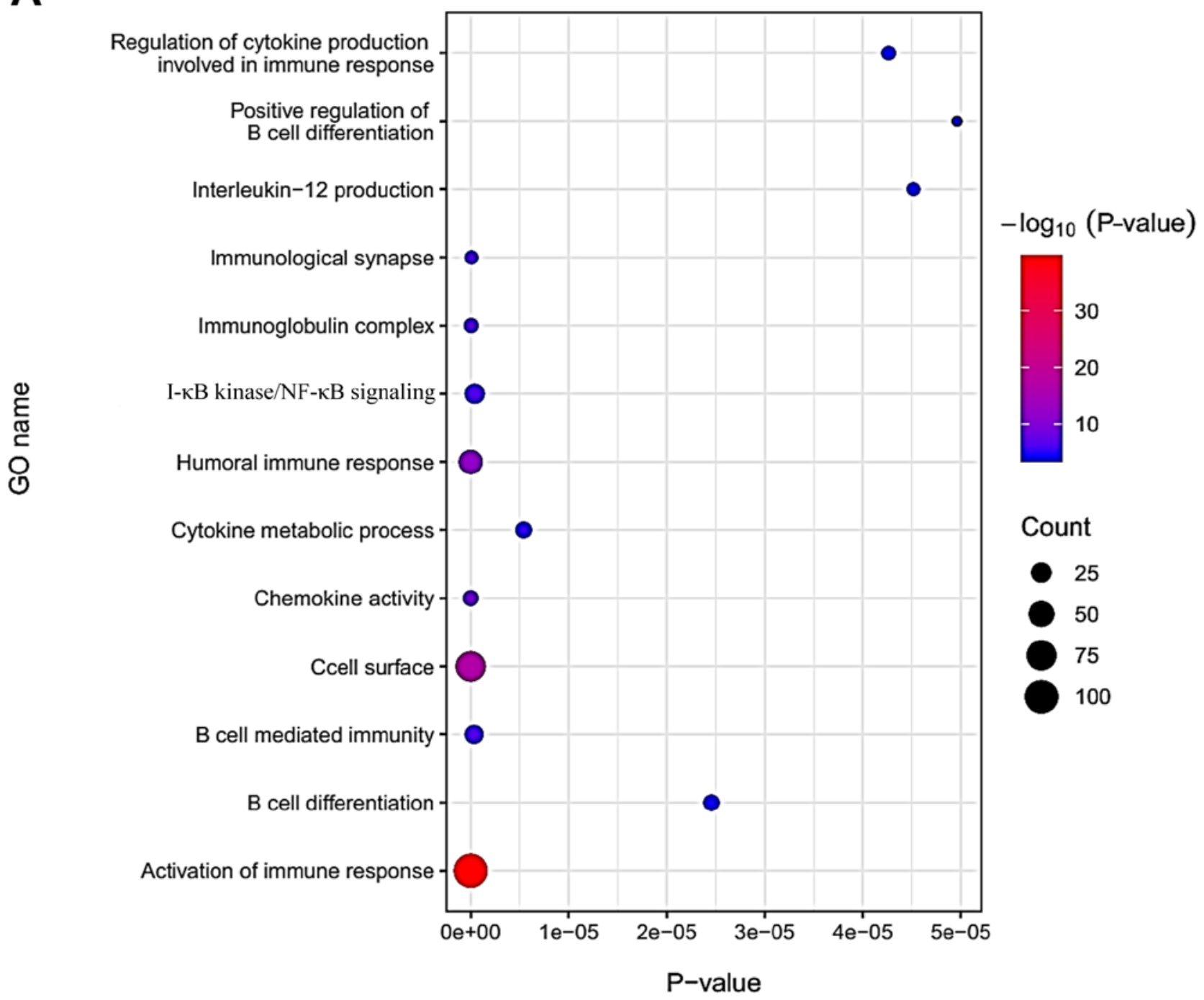

B

KEGG enrichment analysis of upregulated gene

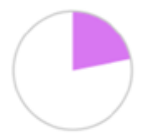

Osteoclast

differentiation

NF-кB signaling pathway

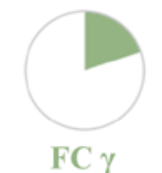

R-mediated

phagocytosis

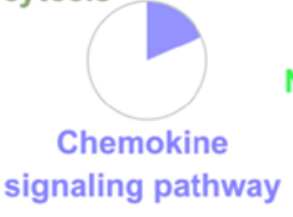

Natural killer cell

mediated

cytotoxicity

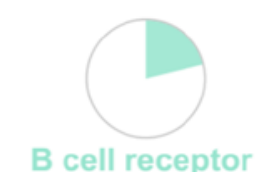

signaling pathway

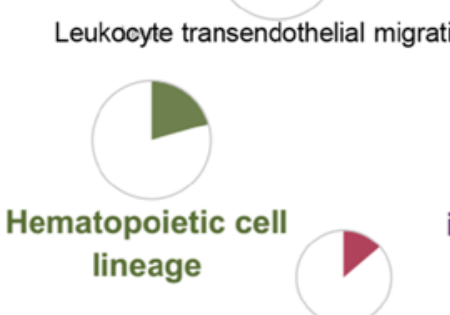

Cell adhesion molecules (CAMs)

Figure 2. Enrichment analysis of upregulated genes. (A) GO enrichment analysis of upregulated genes. (B) KEGG enrichment analysis of upregulated genes. GO, Gene Ontology; KEGG, Kyoto Encyclopedia of Genes and Genomes. 
A

\section{Gene Oncology enrichment analysis of downregulated gene}

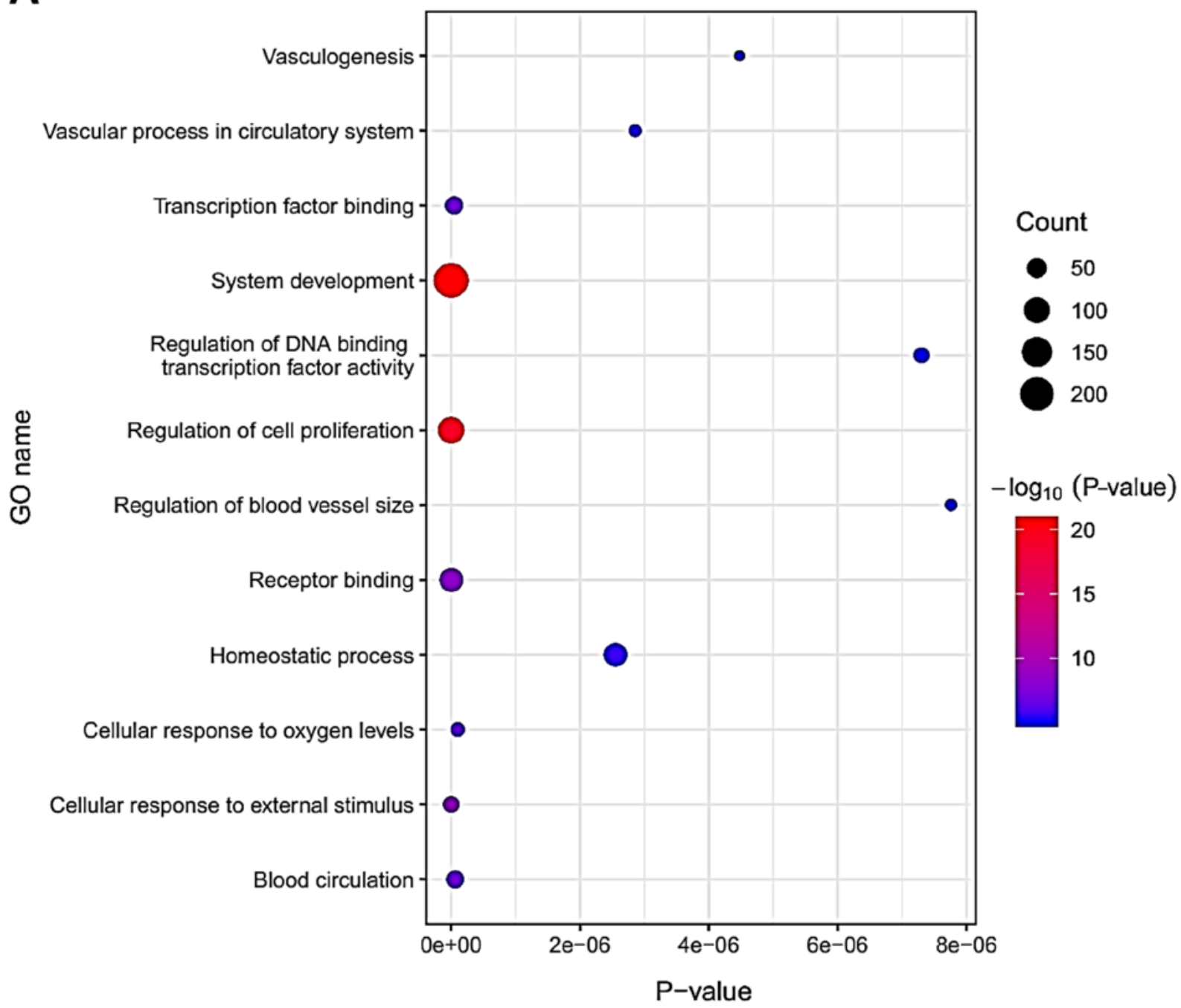

B

KEGG enrichment analysis of downregulated gene

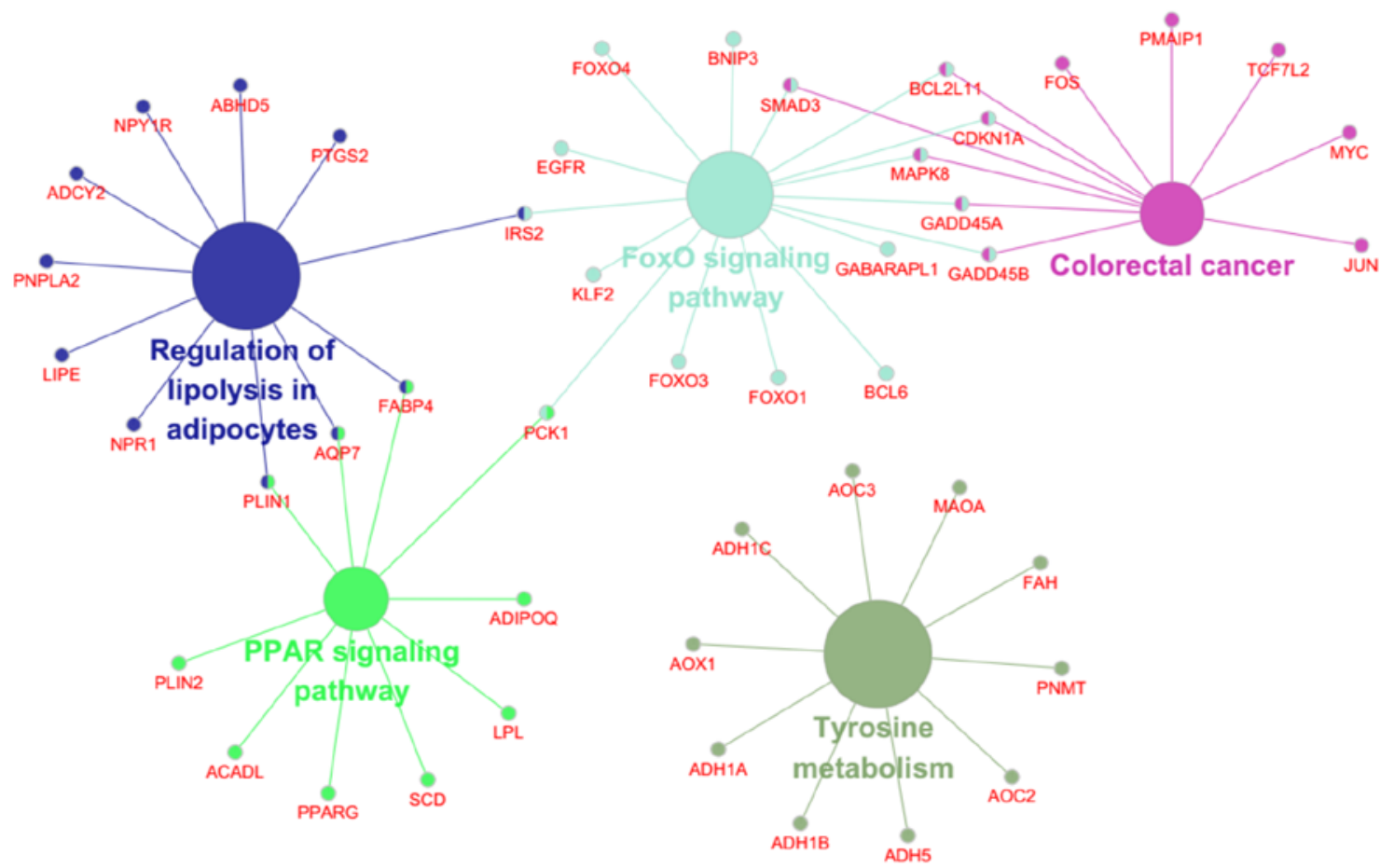

Figure 3. Enrichment analysis of downregulated genes. (A) GO enrichment analysis of downregulated genes. (B) KEGG enrichment analysis of downregulated genes. GO, Gene Ontology; KEGG, Kyoto Encyclopedia of Genes and Genomes. 

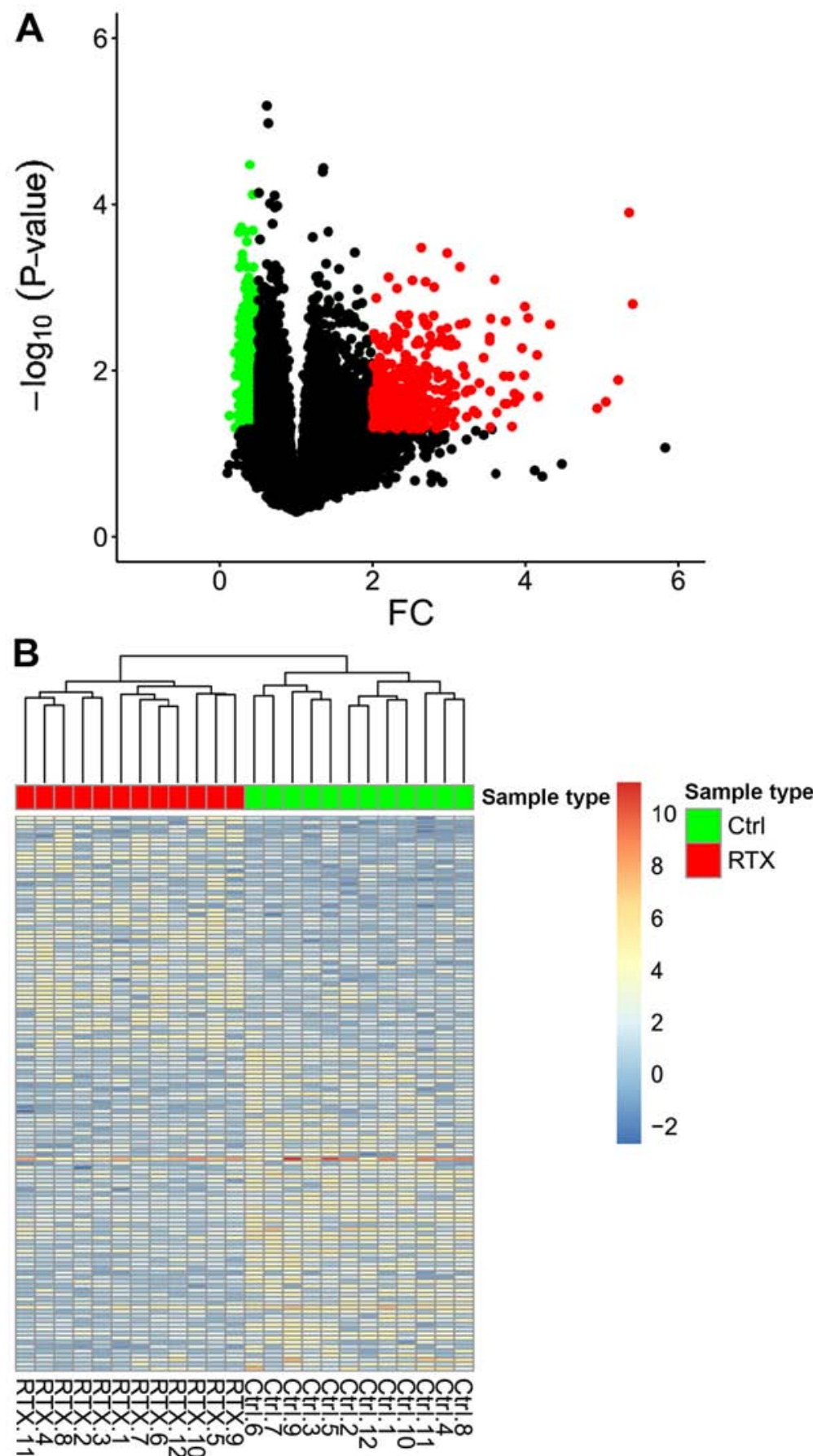

Figure 4. Analysis of differentially expressed genes between synovial tissue samples from patients with rheumatoid arthritis before and after treatment with RTX. (A) Volcano plots of genes between synovial tissue samples from patients with rheumatoid arthritis before and after treatment with RTX. The red and green dots indicate the upregulated and downregulated genes, respectively. The black dots correspond to gene expression with $\mid \log _{2} \mathrm{FCl}<1$. The $y$-axis represents false discovery rate and the x-axis denotes the value of FC. (B) Hierarchical cluster analysis of expression profiles of differentially expressed genes before and after treatment with rituximab. RTX, rituximab; Ctrl, control; FC, fold-change.

presented in Fig. 7A. Briefly, a total of 872 TCM compounds demonstrating the highest docking score with LILRB1 were screened using the high-throughput method, and 43 of them were selected. Subsequently, 5 candidate TCM compounds were obtained via precise docking. Among them, kaempferol 3-O- $\beta$-D-glucosyl-(1 $\rightarrow 2)-\beta-D$-glucoside exhibited the highest binding capacity. The interaction between LILRB1 and kaempferol 3-O- $\beta$-D-glucosyl- $(1 \rightarrow 2)-\beta-D-g$ lucoside is shown in Fig. 7B. The key amino acid residues Cys144, Arg72, Pro90, Val15 and Glu136 of the kaempferol
3-O- $\beta$-D-glucosyl- $(1 \rightarrow 2)-\beta$-D-glucoside binding site interacted with LILRB1 via hydrogen bonds (Fig. 7B). The $3 \mathrm{D}$ conformation of the interaction and surface binding of kaempferol 3-O- $\beta$-D-glucosyl- $(1 \rightarrow 2)-\beta$-D-glucoside and LILRB1 with other proteins were analyzed in order to identify additional protein interactions (Fig. 8A and B). The aforementioned results indicated that kaempferol 3 -O- $\beta$-D-glucosyl- $(1 \rightarrow 2)-\beta$-D-glucoside may decrease the biological activity of LILRB1 by inhibiting its active center, thereby exhibiting therapeutic effects on RA. 
A

Enrichment plot: GO_LEUKOCYTE_MIGRATION

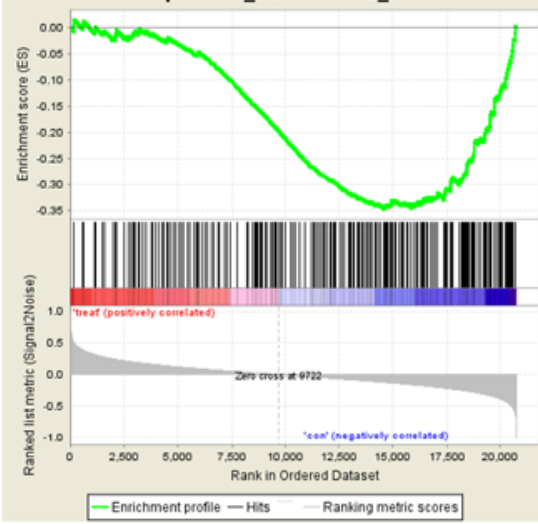

Enrichment plot:

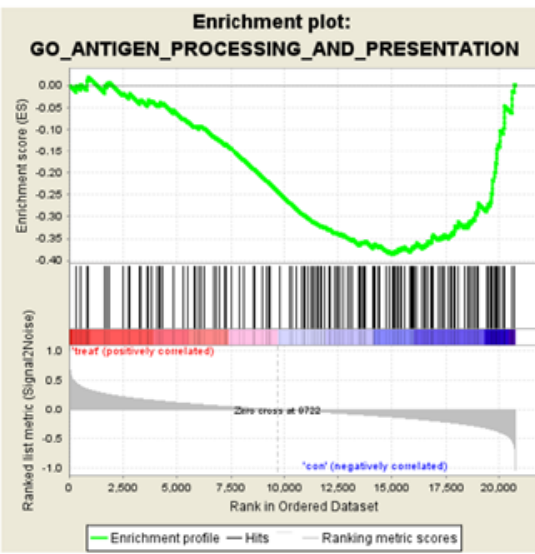

Enrichment plot:

GO_POSITIVE_REGULATION_OF_DENDRITE_DEVELOP

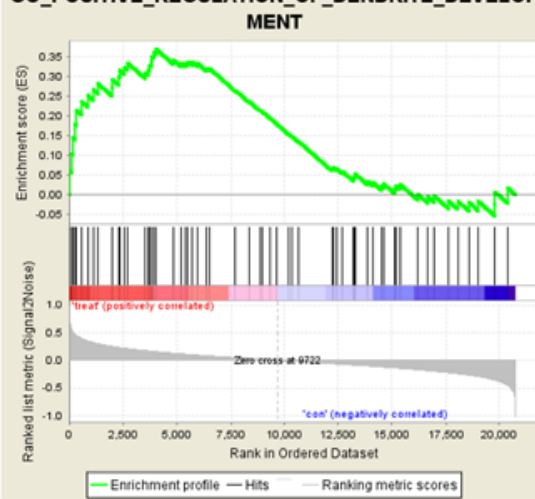

Enrichment plot: GO_IMMUNE_EFFECTOR_PROCESS

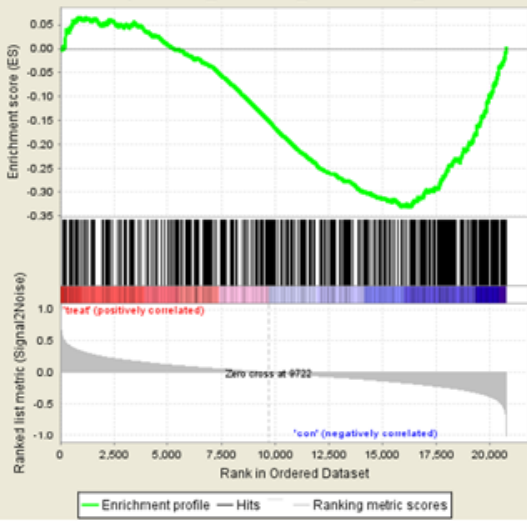

Enrichment plot:

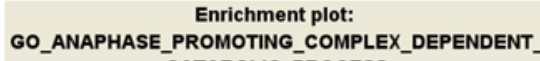
CATABOLIC_PROCESS

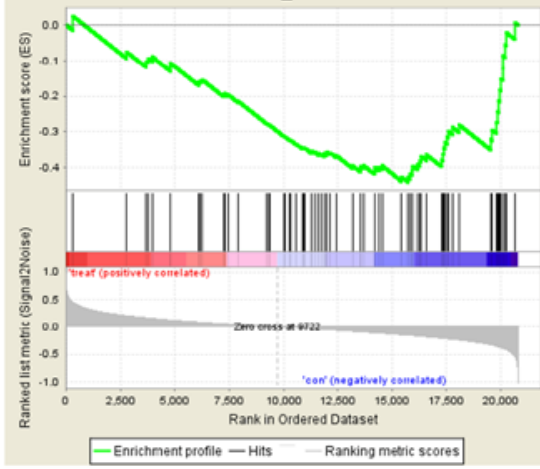

Drichment plot:

GO_CEREBRAL_CORTEX_DEVELOPMENT
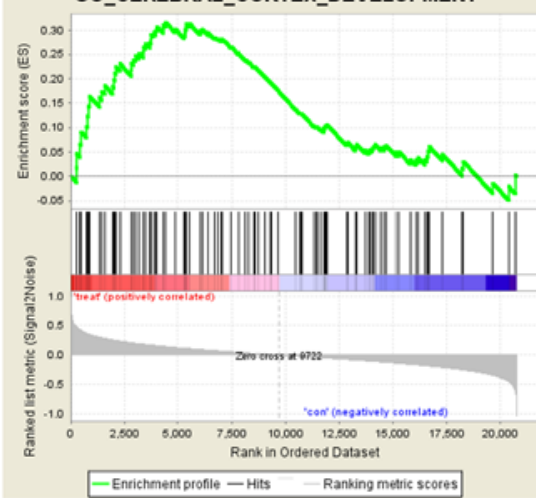

B
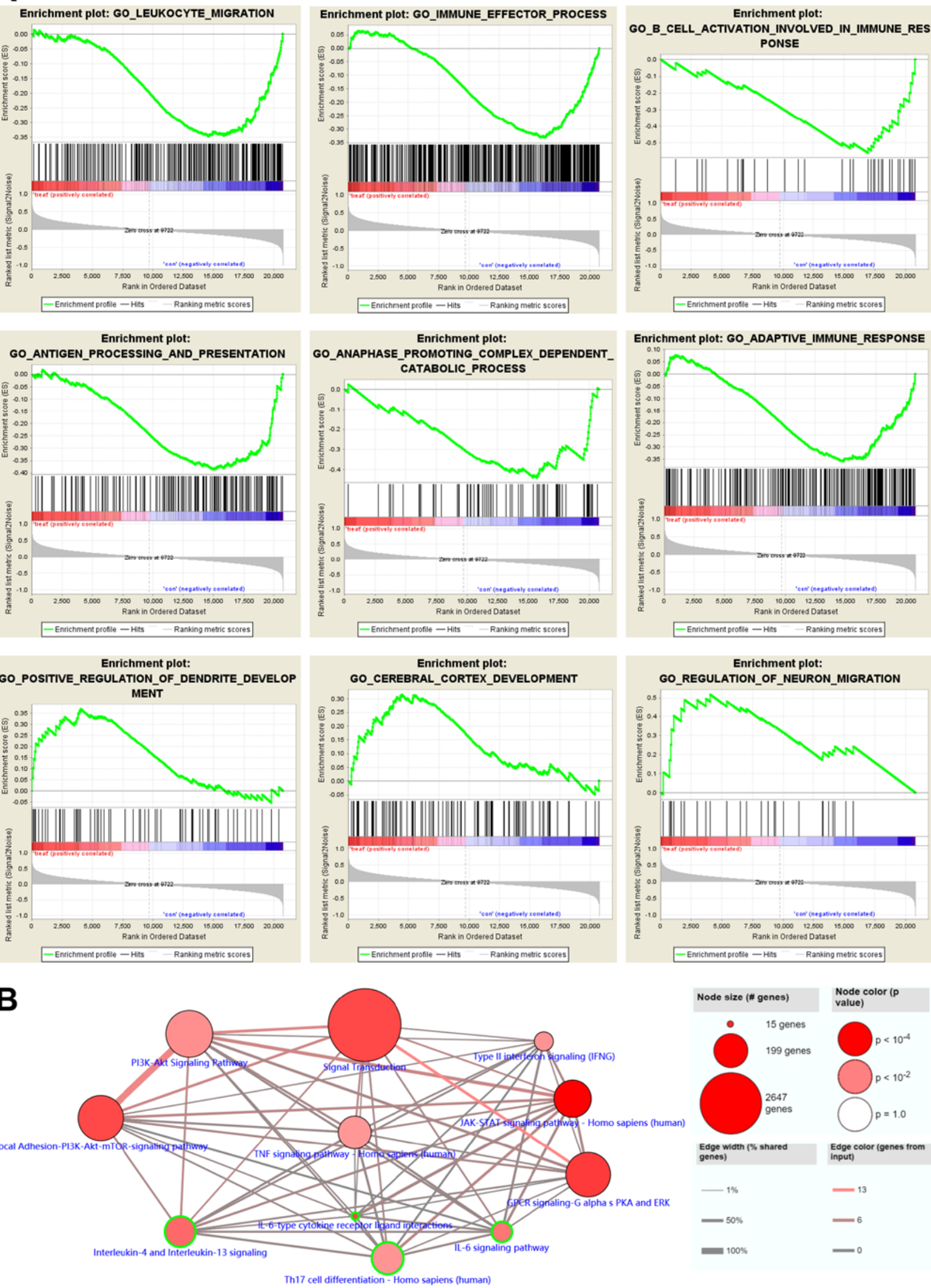

GO_REGULATION_OF_NEURON_MIGRATION
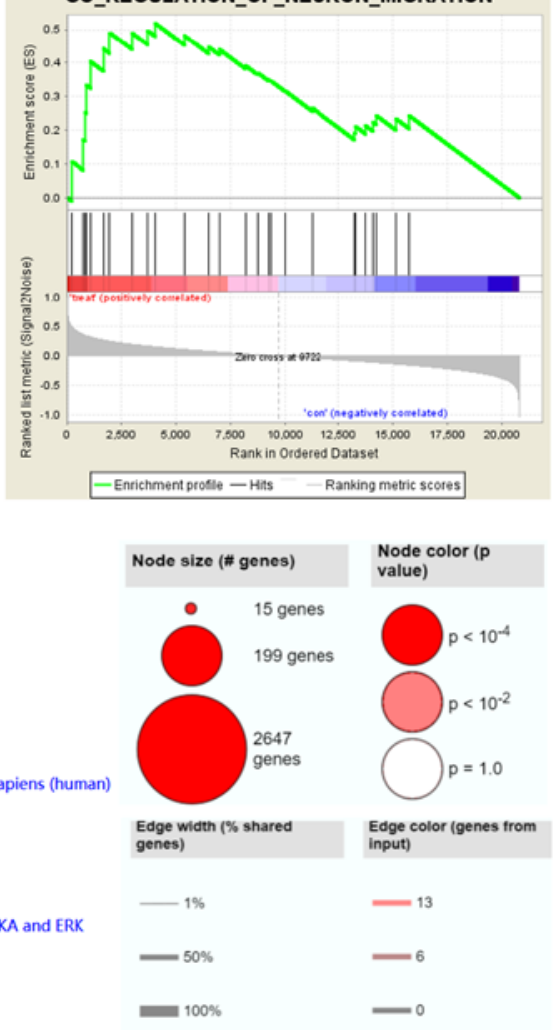

Figure 5. Function and pathway analyses of differentially expressed genes between synovial tissue samples from patients with rheumatoid arthritis before and after treatment with RTX. (A) Gene set enrichment analysis of rituximab in rheumatoid arthritis treatment. (B) Kyoto Encyclopedia of Genes and Genomes pathway enrichment analysis of downregulated genes after treatment with rituximab. GO, Gene Ontology; GPCR, G protein-coupled receptor; IL, interleukin; JAK, Janus kinase; PKA, protein kinase A; Th17, T helper 17 cell; TNF, tumor necrosis factor. 


\section{A}

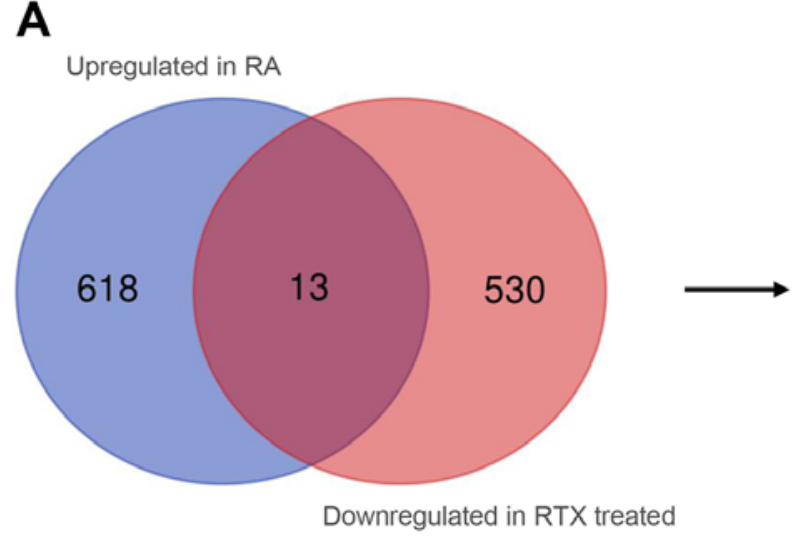

\begin{tabular}{|c|}
\hline LILRB4 \\
\hline WWC1 \\
\hline APOBEC3A \\
\hline LILRB1 \\
\hline PLA2G2D \\
\hline CLEC7A \\
\hline GBP1 \\
\hline IGHA1 \\
\hline JMJD4 \\
\hline ST6GAL1 \\
\hline NUP43 \\
\hline PASK \\
\hline KLRD1 \\
\hline
\end{tabular}

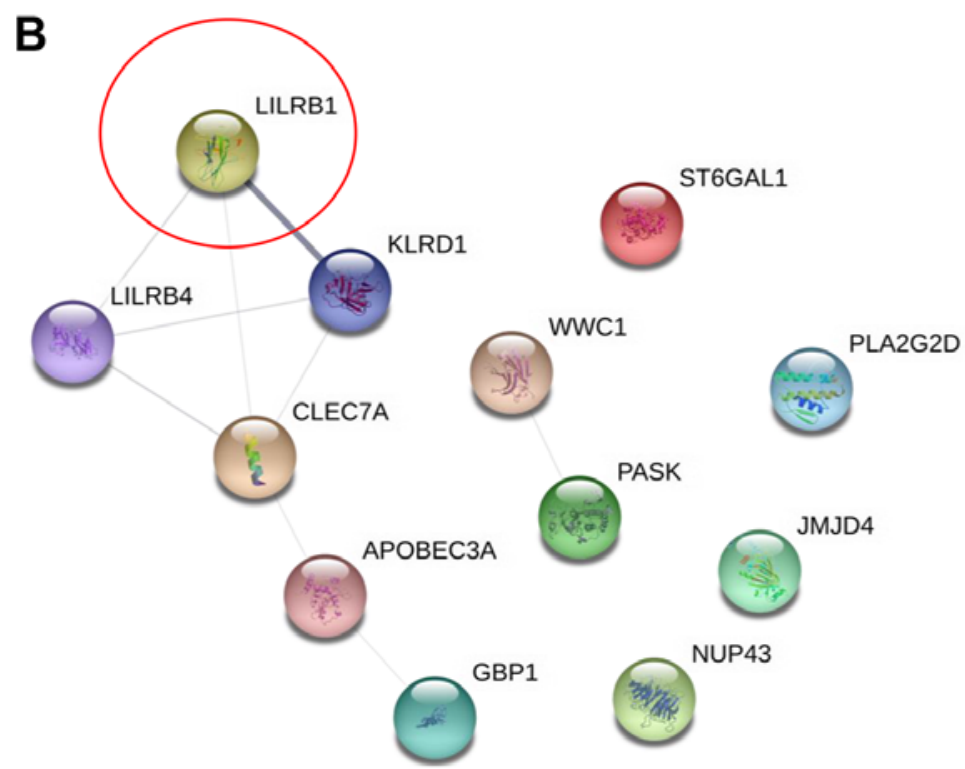

Figure 6. Identification of key genes. (A) Wayne analysis of upregulated genes in RA and downregulated genes after RTX treatment. (B) Protein-protein interaction network analysis of key genes. RA, rheumatoid arthritis; RTX, rituximab.

\section{Discussion}

Immune dysregulation has been implicated in the pathogenesis of RA through the anti-immunoglobulin $\mathrm{G}$ antibodies (35). Although several mechanisms regarding the role of immune regulation in RA pathology have been proposed (36), the exact mechanism should be further investigated. Therefore, in the present study, the aim was to delineate the underlying molecular mechanisms and identify the key regulatory genes involved in the developmental process of RA.

As gene expression profiles may reflect disease status (37), in the present study the expression of DEGs was compared in normal, RA control and RTX-treated tissues selected from the GO database. The DEGs functional analysis indicated that RA was associated with the induction of inflammation responses and immune activities. These observations were consistent with the clinical characteristics of the disease. However, following RTX treatment, inflammation and immune-related signaling pathways were widely suppressed. These results were consistently with the previously reported inflammation- and immune-related characteristics of RA pathogenesis (3). The upregulated and downregulated genes in RA and RTX-treated groups, respectively, were subjected to Venn analysis and subsequently 13 selected genes were screened in order to identify the key regulatory genes. Topological analysis was conducted to screen the node genes and LILRB1, a receptor expressed on the membrane of immune cells (10). The analysis demonstrated that LILRB1 exhibited a high degree of connectivity. The results of the present study indicated that LILRB1 may control inflammatory responses and cytotoxicity by inducing a targeted immune response and limiting autoreactivity. In addition, it was hypothesized that upstream regulatory signaling pathways of the downregulated LILRB1 gene could serve as a target of RTX. However its pharmacological mechanisms of action should be further investigated. Thus, the results indicated that LILRB1 was a potential target of RA treatment.

In order to clarify the target activity of LILRB1, potential drugs that target LILRB1 were screened using the TCM libraries. Kaempferol 3-O- $\beta$-D-glucosyl- $(1 \rightarrow 2)-\beta$-D-glucoside showed the strongest targeting activity. Kaempferol, a natural flavonol, has been reported to act as an antioxidant by reducing oxidative stress during the treatment of several diseases (38). However, the precise molecular mechanisms of kaempferol need to be further studied. The results of the present study revealed that kaempferol could bind to the active pocket of 
A

B
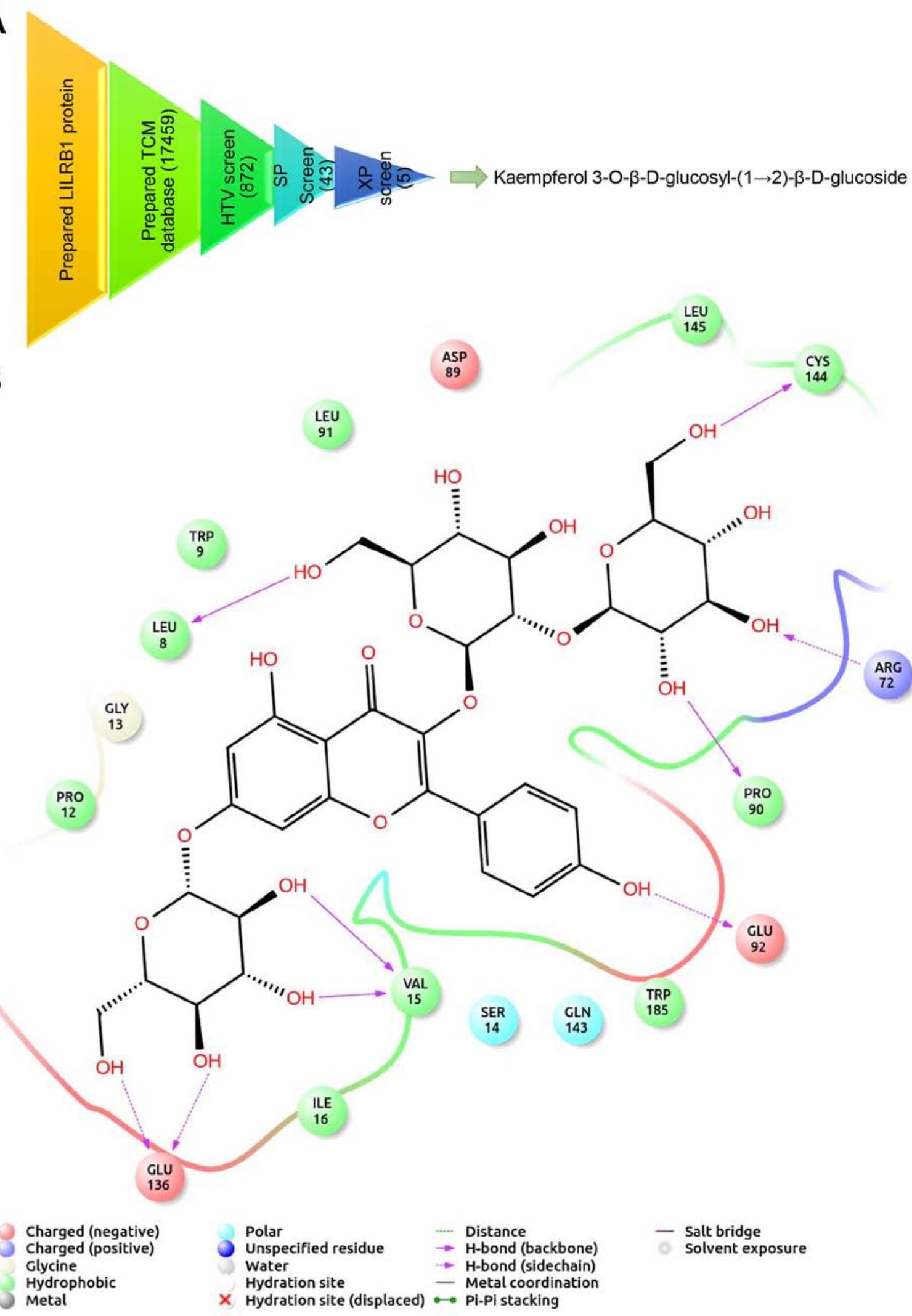

Figure 7. Binding mode of receptor-ligand interaction. (A) Protocol flowchart of LILRB1 inhibitor discovery strategy. (B) 2D combination mode of LILRB1-kaempferol 3-O- $\beta$-D-glucosyl-( $(\rightarrow 2)-\beta$-D-glucoside. LILRB1, leukocyte immunoglobulin-like receptor subfamily B member 1; TCM, Traditional Chinese Medicine; HTV, high throughput virtual; SP, standard precision; XP, extra precision.

LILRB1, suggesting inhibition of the immune response signal transduction via the receptor. Thus, the activity of kaempferol, a potential anti-RA drug, may be mediated via targeting the immune-related receptor. Additional studies investigating the clinical and molecular basis are required to further elucidate the effect of LILRB1 in regulating the pathogenesis of RA 


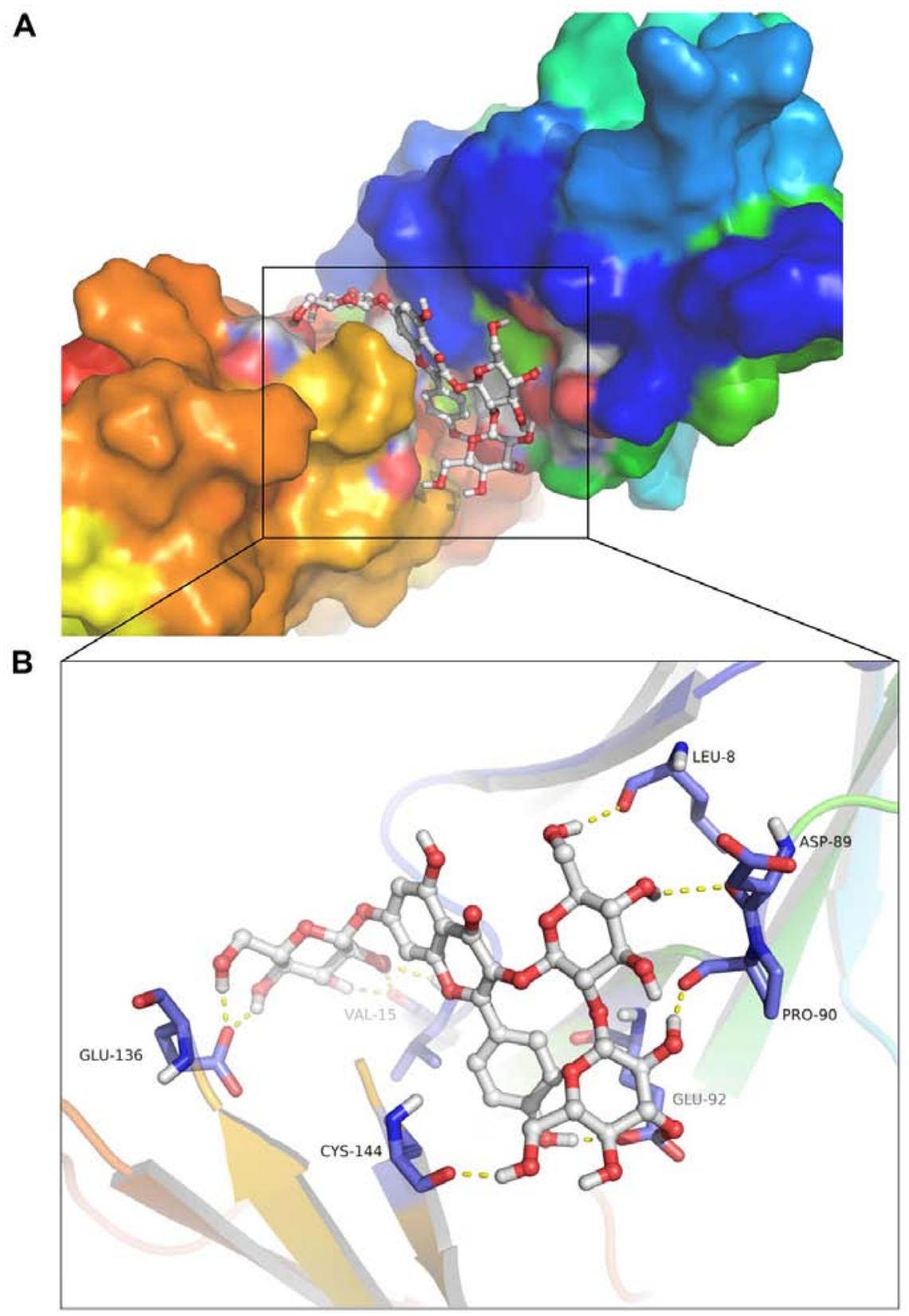

Figure 8. Binding mode of LILRB1-kaempferol 3-O- $\beta$-D-glucosyl-(1 $\rightarrow 2)-\beta$-D-glucoside interaction. (A) Protein surface binding mode of LILRB1-kaempferol 3-O- $\beta$-D-glucosyl-( $(\rightarrow 2)-\beta$-D-glucoside. (B) Cartoon combination mode of LIRNB1-kaempferol 3-O- $\beta$-D-glucosyl-( $1 \rightarrow 2)-\beta$-D-glucoside. LILRB1, leukocyte immunoglobulin-like receptor subfamily B member 1.

and to demonstrate the therapeutic value of kaempferol in RA treatment.

In conclusion, this study may enhance the understanding of RA development processes, and suggested that kaempferol could be a potential novel and effective drug in RA treatment in clinical practice.

\section{Acknowledgements}

Not applicable.

\section{Funding}

This work was supported by Tianjin Science and Technology Commission (grant no. 16ZXMJSY00220).

\section{Availability of data and materials}

The datasets used and/or analyzed during the present study are available from the corresponding author upon reasonable request.

\section{Authors' contributions}

CK conceived and designed the present study. YS and WQ performed the bioinformatics analyses. CK and WQ wrote the manuscript.

\section{Ethics approval and consent to participate}

Not applicable. 


\section{Patient consent for publication}

Not applicable.

\section{Competing interests}

The authors declare that they have no competing interests.

\section{References}

1. Falconer J, Murphy AN, Young SP, Clark AR, Tiziani S, Guma M and Buckley CD: Review: Synovial cell metabolism and chronic inflammation in rheumatoid arthritis. Arthritis Rheumatol 70 984-999, 2018

2. Nakajima A, Aoki Y, Sonobe M, Takahashi H, Saito M, Terayama $\mathrm{K}$ and Nakagawa K: Radiographic progression of large joint damage in patients with rheumatoid arthritis treated with biological disease-modifying anti-rheumatic drugs. Mod Rheumatol 26: 517-521, 2016.

3. Firestein GS and McInnes IB: Immunopathogenesis of rheumatoid arthritis. Immunity 46: 183-196, 2017.

4. Arend WP and Firestein GS: Pre-rheumatoid arthritis: Predisposition and transition to clinical synovitis. Nat Rev Rheumatol 8: 573-586, 2012.

5. Firestein GS: Evolving concepts of rheumatoid arthritis. Nature 423: 356-361, 2003.

6. Singh JA, Saag KG, Bridges SL Jr, Akl EA, Bannuru RR, Sullivan MC, Vaysbrot E, McNaughton C, Osani M, Shmerling RH, et al: 2015 American college of rheumatology guideline for the treatment of rheumatoid arthritis. Arthritis Rheumatol 68: 1-26, 2016.

7. McInnes IB and Schett G: Pathogenetic insights from the treatment of rheumatoid arthritis. Lancet 389: 2328-2337, 2017.

8. Lopez-Olivo MA, Amezaga Urruela M, McGahan L, Pollono EN and Suarez-Almazor ME: Rituximab for rheumatoid arthritis Cochrane Database Syst Rev 1: CD007356, 2015.

9. Smolen JS and Aletaha D: Rheumatoid arthritis therapy reappraisal: Strategies, opportunities and challenges. Nat Rev Rheumatol 11: 276-289, 2015.

10. Young NT, Waller EC, Patel R, Roghanian A, Austyn JM and Trowsdale J: The inhibitory receptor LILRB1 modulates the differentiation and regulatory potential of human dendritic cells Blood 111: 3090-3096, 2008

11. Wang X, Xu Z, Ren X, Chen X, Wei J, Lin W, Li Z, Ou C, Gong Z and Yan Y: Function of low ADARB1 expression in lung adenocarcinoma. PLoS One 14: e0222298, 2019.

12. Yan Y, Xu Z, Hu X, Qian L, Li Z, Zhou Y, Dai S, Zeng S and Gong Z: SNCA is a functionally low-expressed gene in lung adenocarcinoma. Genes (Basel) 9: E16, 2018.

13. Woetzel D, Huber R, Kupfer P, Pohlers D, Pfaff M, Driesch D, Häupl T, Koczan D, Stiehl P, Guthke R and Kinne RW: Identification of rheumatoid arthritis and osteoarthritis patients by transcriptome-based rule set generation. Arthritis Res Ther 16: R84, 2014.

14. Edgar R, Domrachev M and Lash AE: Gene Expression Omnibus: NCBI gene expression and hybridization array data repository. Nucleic Acids Res 30: 207-210, 2002

15. Lauwerys BR, Hernández-Lobato D, Gramme P, Ducreux J, Dessy A, Focant I, Ambroise J, Bearzatto B, Nzeusseu Toukap A, Van den Eynde BJ, et al: Heterogeneity of synovial molecular patterns in patients with arthritis. PLoS One 10: e0122104, 2015

16. Perry M: Heatmaps: Flexible Heatmaps for Functional Genomics and Sequence Features. R package version 1.12.0, 2020. 10.18129/B9.bioc.heatmaps.

17. Ritchie ME, Phipson B, Wu D, Hu Y, Law CW, Shi W and Smyth GK: limma powers differential expression analyses for RNA-sequencing and microarray studies. Nucleic Acids Res 43: e47, 2015 .

18. Ashburner M, Ball CA, Blake JA, Botstein D, Butler H, Cherry JM, Davis AP, Dolinski K, Dwight SS, Eppig JT, et al: Gene ontology: Tool for the unification of biology. The gene ontology consortium. Nat Genet 25: 25-29, 2000.

19. The Gene Ontology Consortium: The gene ontology resource: 20 years and still GOing strong. Nucleic Acids Res 47: D330-D338, 2019.
20. Kanehisa M, Sato Y, Furumichi M, Morishima K and Tanabe M New approach for understanding genome variations in KEGG. Nucleic Acids Res 47: D590-D595, 2019.

21. Bindea G, Mlecnik B, Hackl H, Charoentong P, Tosolini M, Kirilovsky A, Fridman WH, Pagès F, Trajanoski Z and Galon J: ClueGO: A Cytoscape plug-in to decipher functionally grouped gene ontology and pathway annotation networks. Bioinformatics 25: 1091-1093, 2009.

22. Bindea G, Galon J and Mlecnik B: CluePedia Cytoscape plugin: Pathway insights using integrated experimental and in silico data. Bioinformatics 29: 661-663, 2013.

23. Subramanian A, Kuehn H, Gould J, Tamayo P and Mesirov JP: GSEA-P: A desktop application for gene set enrichment analysis. Bioinformatics 23: 3251-3253, 2007.

24. Herwig R, Hardt C, Lienhard M and Kamburov A: Analyzing and interpreting genome data at the network level with ConsensusPathDB. Nat Protoc 11: 1889-1907, 2016.

25. Kamburov A, Wierling C, Lehrach $\mathrm{H}$ and Herwig R: ConsensusPathDB-a database for integrating human functional interaction networks. Nucleic Acids Res 37: D623-628, 2009.

26. Snel B, Lehmann G, Bork P and Huynen MA: STRING: A web-server to retrieve and display the repeatedly occurring neighbourhood of a gene. Nucleic Acids Res 28: 3442-3444, 2000.

27. Sanderson K: Databases aim to bridge the East-West divide of drug discovery. Nat Med 17: 1531, 2011.

28. Halgren TA, Murphy RB, Friesner RA, Beard HS, Frye LL, Pollard WT and Banks JL: Glide: A new approach for rapid, accurate docking and scoring. 2. Enrichment factors in database screening. J Med Chem 47: 1750-1759, 2004.

29. Friesner RA, Banks JL, Murphy RB, Halgren TA, Klicic JJ, Mainz DT, Repasky MP, Knoll EH, Shelley M, Perry JK, et al: Glide: A new approach for rapid, accurate docking and scoring. 1. Method and assessment of docking accuracy. J Med Chem 47: $1739-1749,2004$

30. Nair SK and Burley SK: X-ray structures of Myc-Max and Mad-Max recognizing DNA. Molecular bases of regulation by proto-oncogenic transcription factors. Cell 112: 193-205, 2003.

31. Gupta S and Bajaj AV: Extra precision glide docking, free energy calculation and molecular dynamics studies of 1,2-diarylethane derivatives as potent urease inhibitors. J Mol Model 24: 261, 2018.

32. Friesner RA, Murphy RB, Repasky MP, Frye LL, Greenwood JR, Halgren TA, Sanschagrin PC and Mainz DT: Extra precision glide: Docking and scoring incorporating a model of hydrophobic enclosure for protein-ligand complexes. J Med Chem 49: 6177-6196, 2006.

33. Zhong W, Liu P, Zhang Q, Li D and Lin J: Structure-based QSAR, molecule design and bioassays of protease-activated receptor 1 inhibitors. J Biomol Struct Dyn 35: 2853-2867, 2017.

34. Yan Y, Su W, Zeng S, Qian L, Chen X, Wei J, Chen N, Gong Z and $\mathrm{Xu} \mathrm{Z}$ : Effect and mechanism of tanshinone I on the radiosensitivity of lung cancer cells. Mol Pharm 15: 4843-4853, 2018.

35. Janossy G, Panayi G, Duke O, Bofill M, Poulter LW and Goldstein G: Rheumatoid arthritis: A disease of T-lymphocyte/macrophage immunoregulation. Lancet 2 : 839-842, 1981.

36. Catrina AI, Joshua V, Klareskog L and Malmström V: Mechanisms involved in triggering rheumatoid arthritis. Immunol Rev 269: 162-174, 2016.

37. Barrett T, Suzek TO, Troup DB, Wilhite SE, Ngau WC, Ledoux P, Rudnev D, Lash AE, Fujibuchi W and Edgar R: NCBI GEO: Mining millions of expression profiles-database and tools. Nucleic Acids Res 33: D562-566, 2005.

38. Kashyap D, Sharma A, Tuli HS, Sak K, Punia S and Mukherjee TK: Kaempferol-A dietary anticancer molecule with multiple mechanisms of action: Recent trends and advancements. J Funct Foods 30: 203-219, 2017.

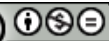

This work is licensed under a Creative Commons Attribution-NonCommercial-NoDerivatives 4.0 International (CC BY-NC-ND 4.0) License. 